\title{
ASPECTOS CONTEXTUAIS SOBRE O GERENCIAMENTO DO CUIDADO DE ENFERMAGEM À CRIANÇA COM DOR ONCOLÓGICA CRÔNICA ${ }^{1}$
}

\author{
Thiago Privado da Silva², Laura Johanson da Silva3 , Maria José Carvalho Ferreira ${ }^{4}$, Ítalo Rodolfo Silva ${ }^{5}$, Benedita \\ Maria Rêgo Deusdará Rodrigues ${ }^{6}$, Joséte Luzia Leite ${ }^{7}$
}

\begin{abstract}
${ }^{1}$ Artigo extraído da Tese - Gerenciamento do cuidado de enfermagem à criança hospitalizada com dor oncológica crônica, apresentada ao Programa de Pós-Graduação em Enfermagem da Escola de Enfermagem Anna Nery (EEAN), da Universidade Federal do Rio de Janeiro (UFRJ), em 2016.

${ }^{2}$ Doutor em Enfermagem. Professor do Curso de Enfermagem da UFRJ - Campus Macaé. Macaé, Rio de Janeiro, Brasil. E-mail: thiagopsilva87@gmail.com

${ }^{3}$ Doutora em Enfermagem. Professora da Universidade Federal do Estado do Rio de Janeiro (UNIRIO). Rio de Janeiro, Rio de Janeiro, Brasil. E-mail: lauraenfaunirio@gmail.com

${ }^{4}$ Mestre em Enfermagem. Tenente da Marinha do Brasil. Rio de Janeiro, Rio de Janeiro, Brasil. E-mail: maria.jcarvalho@live.com

${ }^{5}$ Doutor em Enfermagem. Professor do Curso de Enfermagem e Obstetrícia da UFRJ - Campus Macaé. Macaé, Rio de Janeiro, Brasil. E-mail: enf.italo@hotmail.com

${ }^{6}$ Doutora em Enfermagem. Professora da Faculdade de Enfermagem da Universidade do Estado do Rio de Janeiro. Rio de Janeiro, Rio de Janeiro, Brasil. E-mail: benedeusdara@gmail.com

${ }^{7}$ Doutora em Enfermagem. Professora do Programa de Pós-Graduação em Enfermagem da EEAN/UFRJ. Rio de Janeiro, Rio de Janeiro, Brasil. E-mail: joluzia@gmail.com
\end{abstract}

\section{RESUMO}

Objetivo: discutir os aspectos contextuais relacionados ao gerenciamento do cuidado de enfermagem à criança hospitalizada com dor oncológica crônica.

Método: pesquisa de abordagem qualitativa, desenvolvida com base no referencial metodológico da Teoria Fundamentada em Dados e no referencial teórico do Pensamento Complexo, a partir de Edgar Morin. A coleta de dados ocorreu por meio da entrevista semiestruturada e da observação não participante. Os 21 participantes da pesquisa foram organizados em três grupos amostrais: o primeiro foi composto por sete enfermeiros; o segundo foi constituído por sete técnicos de enfermagem e o terceiro foi formado por sete profissionais da equipe multiprofissional de saúde. Os resultados da pesquisa foram validados por cinco examinadores, dos quais três apresentavam expertise no método de pesquisa.

Resultados: a categoria intitulada "Desvelando aspectos contextuais sobre o gerenciamento do cuidado de enfermagem à criança com dor oncológica crônica" revelou por meio de suas subcategorias que a hospitalização dessa criança é longa, marcada por mudanças na dinâmica familiar, perdas e limitações impostas pela doença e/ou tratamento. O gerenciamento do cuidado de enfermagem é complexo, realizado por meio do trabalho em equipe e da demanda um planejamento que valorize as múltiplas necessidades da criança e do familiar.

Conclusão: os resultados apontam para a necessidade do profissional de enfermagem contextualizar suas relações de cuidado, a partir da valorização de aspectos que transcendem a dimensão biológica da criança hospitalizada com dor oncológica crônica.

DESCRITORES: Enfermagem pediátrica. Cuidado da criança. Criança hospitalizada. Dor crônica. Neoplasias.

\section{CONTEXTUAL ASPECTS RELATED TO NURSING CARE MANAGEMENT OF THE CHILD WITH CHRONIC CANCER PAIN}

\begin{abstract}
Objective: to discuss the contextual aspects related to nursing care management of hospitalized children with chronic cancer pain.

Method: a qualitative research developed and based on the methodological framework of the Grounded Theory and the Complex Thought theoretical framework of Edgar Morin. Data were collected through a semi-structured interview and non-participant observation. The 21 participants of the research were organized into three sample groups: the first was composed of seven nurses; the second was made up of seven nurse technicians and the third was formed by seven professionals from the multiprofessional health team. The results of the research were validated by five examiners, three of whom were experts in the research method. Results: the "Unveiling contextual aspects of nursing care management of children with chronic cancer pain" category and subcategories revealed that hospitalization of these children is long and is marked by changes in family dynamics, losses and limitations imposed by the disease and / or treatment. Nursing care management is complex, performed through teamwork and requires planning that values the various needs of the child and the family.

Conclusion: the results show that nursing professionals must contextualize their care relationships, by putting value on the aspects that transcend the biological dimension of hospitalized children with chronic cancer pain.

DESCRIPTORS: Pediatric nursing. Child care. Hospitalized child. Chronic pain. Neoplasms.
\end{abstract}




\title{
ASPECTOS CONTEXTUALES SOBRE EL GERENCIAMIENTO DEL CUIDADO DE LA ENFERMERÍA PARA EL NIÑO CON DOLOR ONCOLÓGICO CRÓNICO ${ }^{1}$
}

\begin{abstract}
RESUMEN
Objetivo: discutir los aspectos contextuales relacionados al gerenciamiento del cuidado de enfermería para el niño hospitalizado con dolor oncológico crónico.

Método: investigación de abordaje cualitativo y desarrollado con base en el referencial metodológico de la Teoría Fundamentada en Datos y en el referente teórico del Pensamiento Complejo a partir de Edgar Morin. La recolección de datos ocurrió por medio de la entrevista semiestructurada y de la observación no participante. Los 21 participantes de la investigación fueron organizados en tres grupos de muestras: el primero fue compuesto por siete enfermeros; el segundo fue constituido por siete técnicos de enfermería y el tercero fue formado por siete profesionales del equipo multiprofesional de salud. Los resultados de la investigación fueron validados por cinco examinadores y tres de ellos mostraron experiencia en el método de investigación.

Resultados: la categoría titulada "Esclareciendo los aspectos contextuales sobre el gerenciamiento del cuidado de la enfermería para el niño con dolor oncológico crónico" rebeló, por medio de sus subcategorías, que la hospitalización de ese niño es larga, marcada por cambios en la dinámica familiar, pérdidas y limitaciones impuestas por la enfermedad y/o tratamiento. El gerenciamiento del cuidado de la enfermería es complejo y realizado por medio del trabajo en equipo y de la demanda de un planeamiento que valorice las múltiples necesidades del niño y del familiar.
\end{abstract}

Conclusión: los resultados señalan la necesidad del profesional de enfermería de contextualizar sus relaciones de cuidado a partir de la valorización de aspectos que transcienden la dimensión biológica del niño hospitalizado y con dolor oncológico crónico.

DESCRIPTORES: Enfermería pediátrica. Cuidado del niño. Niño hospitalizado. Dolor crónico. Neoplasias.

\section{INTRODUÇÃO}

No Brasil, o câncer infanto-juvenil (crianças e adolescentes de zero até 19 anos) é considerado raro quando comparado com os tumores do adulto, pois corresponde entre $2 \%$ e $3 \%$ de todos os tumores malignos, destacando-se como a mais importante causa de óbito nos países em desenvolvimento. ${ }^{1}$ Entre os tipos de câncer mais comum na infância, estão as leucemias, ${ }^{2}$ que podem ser do tipo linfoide e não linfoide, aguda ou crônica.

Na conjuntura nacional, para o ano de 2016, estimou-se a ocorrência de 12.600 novos casos de câncer em crianças e adolescentes até os 19 anos, sendo as regiões Sudeste e Nordeste as com maiores números de casos novos, 6.050 e 2.750 , respectivamente. ${ }^{1}$ Nos Estados Unidos, a taxa de incidência anual de câncer em crianças e adolescentes é de 186.6 por 1 milhão de crianças entre zero e 19 anos. Esse mesmo país revelou em 2014 a estimativa de 15.780 novos casos de câncer e 1.960 mortes provenientes desta doença entre crianças e adolescentes de zero a 19 anos. $^{3}$

No contexto oncológico pediátrico, a dor se destaca como um dos sintomas mais comum e angustiante, pois gera um importante sofrimento infantil e familiar, como também influencia todo o processo de hospitalização da criança. ${ }^{4-6}$ Em um estudo, ${ }^{4}$ a dor representou $56 \%$ das queixas referidas por crianças com câncer avançado, sendo a cabeça o local mais comum, seguido do abdome, costa, perna e pés. Em conformidade com esse resultado, um outro estudo, ${ }^{6}$ realizado em hospitais na Nigéria, apresentou a dor como um dos principais sintomas vivenciados pela criança em tratamento de câncer. Soma-se a essa condição outros sintomas, a saber: náusea, falta de energia, falta de apetite, perda do cabelo e perda de peso. ${ }^{6}$

Ressalta-se que, nos países em desenvolvimento, onde muitas crianças apresentam o câncer em estado avançado e poucas têm acesso a um tratamento efetivo, a dor está relacionada à progressão da doença. Nos países desenvolvidos, a dor oncológica na infância está relacionada ao tratamento e aos procedimentos diagnósticos e terapêuticos. ${ }^{7} \mathrm{No}$ entanto, admite-se que seja qual for a sua causa, a dor oncológica é sempre um incômodo para a criança, e desse modo, precisa ser avaliada e gerenciada pelos profissionais de enfermagem, no âmbito do trabalho em equipe, de forma correta e imediata, a fim de atenuar seus impactos negativos na qualidade de vida da criança.

A dor oncológica pode se manifestar como aguda ou crônica. A dor aguda surge como resultado de uma lesão tecidual e tende a desaparecer quando o ferimento cicatriza. Por outro lado, a dor crônica é contínua (persistente) ou recorrente (episódica) e persiste além do tempo normal esperado. ${ }^{7}$ Enquanto a dor aguda é geralmente autolimitada, em alguns pacientes, a dor persiste além do tempo de cura esperado (arbitrariamente definido $>3-6$ meses), que é classificado como dor crônica. ${ }^{8}$

A literatura ${ }^{7}$ ressalta que a presença da dor crônica em crianças com doença crônica, como por exemplo, o câncer, pode afetar negativamente vários aspectos da vida infantil, incluindo a prática 
de atividades físicas, frequência escolar, padrão de sono, relações sociais, entre outros aspectos. A esse respeito, estudo ${ }^{5}$ identificou que as crianças que manifestaram dor oncológica crônica apresentaram dificuldades no âmbito extracurricular $(16,7 \%)$, doméstico $(14,6 \%)$, social $(12,6 \%)$, sono $(12,6 \%)$ e acadêmico $(12,5 \%)$.

Isso posto, considera-se importante um gerenciamento do cuidado de enfermagem que contemple a multidimensionalidade da dor oncológica crônica, o sofrimento infantil e familiar, como também os aspectos subjacentes ao processo de hospitalização da criança. Em vista disso, o referencial da complexidade na perspectiva de Edgar Morin, surge como possibilidade para subsidiar uma percepção mais contextualizada sobre a realidade da criança e de sua família, posto que se refere a um pensamento que valoriza a relação inseparável entre o fenômeno e o seu contexto, bem como as inter-relações entre os constituintes heterogêneos intrinsecamente associados, ${ }^{9}$ como é o caso da dor.

Nessa direção, embora a literatura ${ }^{5,10-13}$ sobre a dor oncológica crônica na infância tenha avançado, por meio de pesquisas científicas de abordagem qualitativa e quantitativa, pouco se sabe sobre os aspectos contextuais que permeiam o gerenciamento do cuidado de enfermagem à criança hospitalizada com dor oncológica crônica, ao passo que se observa um foco dos pesquisadores principalmente para as questões relativas à avaliação e ao manejo da dor oncológica crônica. Assim, a compreensão desses aspectos, sob a lente da complexidade, ganha notória relevância, haja vista sua capacidade em influenciar o processo de avaliação e manejo da dor, como também a efetividade do tratamento da criança hospitalizada com câncer. Desse modo, o objetivo desse estudo consiste em discutir, à luz do Pensamento Complexo de Edgar Morin, os aspectos contextuais relacionados ao gerenciamento do cuidado de enfermagem à criança hospitalizada com dor oncológica crônica.

\section{MÉTODO}

Estudo de abordagem qualitativa, realizado com base no referencial metodológico da Grounded Theory, em português, Teoria Fundamentada nos Dados, e no referencial teórico do Pensamento Complexo, na perspectiva de Edgar Morin. O método da Grounded Theory foi usado em virtude de sua capacidade peculiar em produzir conceitos densos e teorizados sobre um fenômeno, ${ }^{14}$ incluindo os processos subjacentes e suas dinâmicas em seu respectivo contexto. ${ }^{15}$ Por outro lado, o uso do Pensamento Com- plexo, na perspectiva de Edgar Morin, é justificado pelo interesse em valorizar a multidimensionalidade da dor oncológica crônica e a inerente complexidade do contexto oncológico pediátrico.

A coleta de dados ocorreu por meio de duas técnicas, a saber: a entrevista semiestruturada e a observação não participante, no período entre agosto de 2014 e junho de 2015. O cenário foi a Unidade de Internação Pediátrica (UIP) de um hospital, localizado na cidade do Rio de Janeiro, Brasil. Esse cenário é constituído por 13 leitos e os principais diagnósticos das crianças hospitalizadas são: leucemia, linfoma e doença falciforme.

Participaram do estudo 21 profissionais de saúde, que preenchiam os seguintes critérios de inclusão: ter experiência mínima de um ano no cuidado à criança oncológica e esse mesmo período de vinculação profissional à instituição. Foram excluídos os profissionais de saúde que estavam de licença ou de férias. Os participantes do estudo foram organizados em grupos amostrais, que se explica pelo recurso da amostragem teórica, ${ }^{15}$ cujo objetivo é buscar pessoas, locais ou fatos que ampliem a possibilidade de descobrir variações entre os conceitos e de tornar densas categorias no que diz respeito às suas propriedades e dimensões. Assim, o primeiro grupo amostral foi composto por sete enfermeiros; o segundo foi constituído por sete técnicos de enfermagem; e o terceiro e último grupo amostral foi formado por sete profissionais da equipe multiprofissional de saúde, sendo duas médicas, duas fisioterapeutas, uma assistente social, uma psicóloga e uma farmacêutica.

As perguntas que nortearam as entrevistas com os enfermeiros foram: como é para você cuidar de uma criança com dor oncológica crônica? Como você percebe a hospitalização da criança com dor oncológica crônica? Quais os significados você atribui ao contexto de cuidados? A análise dos discursos revelou que o contexto de cuidado à criança com dor oncológica crônica é marcado pela necessidade de um trabalho em equipe, cujo objetivo consiste em oferecer à criança e à sua família uma assistência alinhada às suas necessidades multidimensionais. Ademais, o trabalho em equipe foi compreendido como estratégia de ação e condição interveniente das relações que permeiam o gerenciamento do cuidado de enfermagem. Considerando a complexidade do fenômeno exposto, houve a necessidade de expandir a coleta de dados para os demais profissionais de saúde.

Para os participantes do segundo e terceiro grupo amostral, as questões norteadoras foram: 
como você percebe a hospitalização da criança com dor oncológica crônica? Quais os significados você atribui ao contexto de cuidados? A observação não participante foi realizada em cinco ocasiões, durante o período diurno, após análise dos discursos, e totalizou 54 horas. Os conteúdos foram registrados em notas de observação. Ressalta-se que a finalização da coleta de dados em cada grupo amostral foi determinada pelo recurso da saturação teórica, momento em que os novos dados coletados já não estavam alterando em consistência e densidade teórica os conceitos construídos. ${ }^{15}$

$\mathrm{Na}$ Grounded Theory, os dados são coletados e paralelamente analisados. A análise comparativa foi utilizada, pois é uma característica das análises conduzidas por este método de pesquisa. Os dados foram analisados por meio das seguintes etapas de codificações: aberta, axial e seletiva. Na codificação aberta, os dados foram codificados linha por linha, gerando os códigos preliminares que, por sua vez, após serem agrupados por similaridades, deram origem aos códigos conceituais. O agrupamento dos códigos conceituais por similaridades originou as categorias e subcategorias.

Na codificação axial, as categorias foram relacionadas entre si e entre suas subcategorias, a fim de determinar suas propriedades e dimensões. Nesse momento da análise, utilizou-se uma ferramenta analítica denominada de Modelo Paradigmático, que possibilitou a reunião/ordenação/integração das categorias previamente elaboradas, favorecendo o surgimento do fenômeno central do estudo, realizado na etapa de codificação seletiva. Nessa última etapa, também foi realizada a validação dos resultados, ${ }^{15}$ que ocorreu nos meses de setembro e outubro de 2016 e contou com a participação de cinco validadores, sendo três pesquisadores da área da Enfermagem com expertise na Grounded Theory e/ou em pesquisa na área da Gerência em Enfermagem e duas enfermeiras assistenciais do primeiro grupo amostral do presente estudo. Destaca-se que a seleção dos validadores ocorreu por conveniência. Aliado ao processo de codificação, foram realizados memorandos e diagramas que auxiliaram a análise teórica dos dados.

Ressalta-se que a coleta de dados somente foi iniciada após a aprovação do estudo pelo Comitê de Ética em Pesquisa da instituição coparticipante, sob parecer de número 355/14, bem como pelo Comitê de Ética em Pesquisa da instituição proponente, sob Parecer de número 816.736 e CAAE 32795514800005238. Todos os participantes assinaram o Termo de Consentimento Livre e Esclarecido (TCLE).
Considerando as recomendações da Resolução 466/2012 do Conselho Nacional de Saúde do Ministério da Saúde, as falas dos enfermeiros estão identificadas pela letra $\mathrm{E}$, as dos técnicos de enfermagem pela letra $T$, as das médicas pela letra $M$, as da farmacêutica pelas letras FC, as da psicóloga pela letra $\mathrm{P}$, as das fisioterapeutas pelas letras FS e as da assistente social pelas letras AS. Todas estão seguidas por um algarismo que se refere à ordem das entrevistas em cada grupo amostral (E1, T1, M1).

\section{RESULTADOS}

Do total de 21 participantes do estudo, apenas um foi do sexo masculino e compôs o primeiro grupo amostral. Nesse mesmo grupo, o tempo de experiência no cuidado à criança oncológica variou entre um e 13 anos. No segundo grupo amostral, considerando o mesmo item, houve uma variação entre um e dois anos. Já no terceiro grupo amostral, as hematopediatras apresentaram experiência que variou entre três e cinco anos. Por outro lado, as fisioterapeutas, bem como a psicóloga apresentaram experiência de um ano e seis meses no cuidado à referida criança, enquanto que a assistente social referiu apresentar dois anos de atuação nesse mesmo cenário. A farmacêutica que participou da pesquisa referiu não atuar de forma direta com a criança oncológica, haja vista sua responsabilidade em gerenciar a farmácia da instituição. No entanto, ela destacou que há dois anos atuava em equipe com os profissionais de saúde da Unidade de Internação Pediátrica.

Do processo de codificação dos dados, emergiu o fenômeno central - Gerenciamento do cuidado de enfermagem à criança hospitalizada com dor oncológica crônica: uma experiência de múltiplas inter-ações, o qual apresenta como condição contextual a categoria intitulada: Desvelando aspectos contextuais sobre o gerenciamento do cuidado de enfermagem à criança com dor oncológica crônica.

Essa categoria é composta por quatro subcategorias: Apresentando especificidades da instituição de saúde; Compreendendo a hospitalização da criança com dor oncológica crônica; Caracterizando o cuidado de enfermagem à criança com dor oncológica crônica; e Revelando sofrimento familiar.

Em Apresentando especificidades da instituição de saúde, destaca-se a peculiaridade do cenário do estudo, haja vista se tratar de um hospital que se configura como um centro estadual de referência para o cuidado às pessoas com doenças hematológicas. Nesse contexto, a oferta de cursos de 
aperfeiçoamento é frequente e necessária, posto ser um cenário de cuidados altamente especializado.

O pessoal aqui é treinado. É um centro de excelência. Não que a gente seja melhor que outros hospitais, mas a gente tem uma noção diferenciada (T3).

Aqui, eles nos oferecem bastante cursos de aperfeiçoamento. Eles nos dão muita orientação e esses cursos são muito importantes (T5).

Os profissionais de saúde dispõem de um protocolo institucional de dor, o qual foi elaborado para nortear a prática clínica de cuidados à pessoa com dor crônica, seja ela adulto ou criança, oncológica ou não oncológica. Nesse ínterim, dada a relevância de uma prática sistematizada, a avaliação e a reavaliação da dor fazem parte da prescrição médica, sendo realizada em horários regulares.

Aqui, nós temos um protocolo de dor e nós o utilizamos em todos os tipos de dor, seja oncológica ou não oncológica (E1).

A instituição começou a cobrar mais a avaliação da dor e entrou na prescrição médica também. Agora, nós temos horários para checagem, tudo direitinho, de 4/4 horas e eles sempre nos orientam a reavaliar essa dor após uma hora (T4).

A oncologia pediátrica apresenta especificidades relativas às relações de cuidado à criança com dor oncológica crônica, que foi significada como diferente quando comparada com as relações de cuidado estabelecidas com o adulto hospitalizado.

Os profissionais de saúde são bem mais ligados aos pacientes por se tratar de crianças [...] Quando você vê uma criança doente com dor, a sua visão é outra, seu atendimento é outro (FC3).

Na pediatria, a equipe fica mais em cima, ela fica mais preocupada e isso é em qualquer hospital, não somente aqui. Então, há sempre um cuidado maior, as orientações são maiores (FS4).

O gerenciamento da Unidade de Internação Pediátrica é realizado pelo enfermeiro diarista, profissional quem gerencia os recursos humanos de enfermagem e os recursos materiais. Todos os enfermeiros desenvolvem o gerenciamento do cuidado de enfermagem, conforme é exposto a seguir.

O gerenciamento da unidade se baseia em um quadro com um enfermeiro plantonista e quatro técnicos plantonistas, que se dividem no plantão diurno e noturno. Permeando isso, a gente tem uma auxiliar de enfermagem diarista que fica responsável pelo material sob nossa supervisão [...] Nós temos materiais específicos e nós fazemos o pedido através de um sistema de acordo com a demanda da unidade. A gente faz escalas, gerencia cobertura de férias, gerencia cobertura de plantões e também nós gerenciamos o cuidado à criança hematológica (E5).

Em Compreendendo a hospitalização da criança com dor oncológica crônica, ressaltou-se que o tratamento da criança com câncer é longo e marcado por frequentes internações. Nesse sentido, o uso de opioides foi revelado com importante estratégia para o controle da dor oncológica crônica, porém, nem sempre, esses medicamentos proporcionam o alívio necessário, no contexto estudado.

O tratamento é prolongado e tem que estar levando sempre em consulta. Há várias internações durante o tratamento (E6).

Você primeiro começa com a medicação e depois a medicação não está mais surtindo o efeito. Então, você faz outra medicação no intervalo. Vocêf faz uma morfina e daqui a pouco, vocêestá fazendo uma dipirona para tentar amenizar um pouquinho e nem sempre isso faz efeito (E4).

São vários os agentes farmacológicos. Nós iniciamos com a dipirona e, às vezes, ela não faz o efeito desejado. Então, a gente entra com ibuprofeno que também não alivia a dor. Depois, nós passamos para morfina ou tramal (E5).

A dor oncológica crônica foi caracterizada pelos profissionais de enfermagem como forte, intensa e desesperadora, pois gera na criança e no familiar um acentuado sofrimento.

Uma coisa muito interessante é que dificilmente uma criança com dor crônica oncológica dá a nota 10 à sua dor. Ela não dá 10. Ela dá 08, 07, 09, porque ela sabe que a sua dor pode piorar. Muitas delas conhecem o máximo de dor e sabem que a dor pode piorar (E1).

É uma dor desesperadora. Eles têm uma dor muito intensa e vocêpercebe um sofrimento muito grande, tanto na criança como na mãe (E5).

A hospitalização da criança com dor oncológica crônica é marcada por inúmeras limitações e perdas, as quais são apresentadas pelo profissional de saúde a seguir.

São muitas limitações e perdas na internação, como a perda da escola, perda dos amigos, da liberdade que é momentânea, do ir e vir na internação, do brincar (P6).

A limitação do brincar esteve, também, presente nos discursos dos enfermeiros. Por essa razão, o lúdico ganha relevância nas relações de cuidado em pediatria, por se apresentar como uma importante necessidade da criança, que é ameaçada pelo processo de hospitalização.

Elas ficam esperando: 'poxa tia, eu estava esperando alguém para brincar comigo!' Uma vez aconteceu de a criança apertar a campainha e eu fui correndo e perguntei o que tinha acontecido e a criança me disse que não tinha 
nada, ela apenas queria que alguém brincasse com ela, porque a mãe dela estava cansada, estressada (E3).

Essa dor é, às vezes, carência de alguém para brincar (E2).

No contexto oncológico, a cura da doença é algo muito desejado pela equipe multiprofissional de saúde, pelo familiar, bem como pela própria criança, porém, nem sempre, é possível alcançar esse objetivo.

Nós sabemos que muitas doenças a gente vai ou não conseguir curar. A anemia falciforme é crônica e você não vai curar nunca. As leucemias, umas a gente consegue curar, outras nós sabemos que não vamos conseguir curar (M1).

Nesse particular, se compreende na subcategoria Caracterizando o cuidado de enfermagem à criança com dor oncológica crônica que essa atividade se configura como uma experiência complexa para os profissionais de enfermagem, haja vista as repercussões das relações de cuidado na dimensão emocional do profissional, posto que a relação com a dialógica entre doença-saúde e vida-morte apresenta particularidades simbólicas no contexto da saúde da criança.

É muito difícil e falo pela parte emocional mesmo, você ver uma criança sofrendo com dor. Você faz uma medicação que é paliativa. Você acompanha aquele quadro dia após dia. Você sabe que não há mais cura e que é só uma questão de tempo (T5).

Por essa razão, os profissionais de enfermagem referiram a necessidade do profissional de saúde apresentar conhecimento técnico, científico, sensibilidade e um preparo emocional para lidar com o sofrimento infantil e familiar.

Esse preparo tanto técnico, como científico e emocional énecessário porque se vocênão está preparado, você fica perdido e tudo acaba sendo novidade (E7).

Nós temos que ter uma base emocional muito forte no cuidado. É muito complicado [...] Você tem que ter sensibilidade para cuidar da criança (T5).

O cuidado de enfermagem à criança hospitalizada com dor oncológica crônica foi caracterizado como uma experiência desgastante, difícil, permeada por aprendizagem profissional e pessoal.

É desgastante, porque é uma dor desesperadora e a equipe tem que lidar com a mãe, com a criança e com todo um contexto que essa dor causa (E5).

É muito difícil esse cuidado até na avaliação da dor, pela dificuldade das crianças classificarem sua dor, mas você aprende muito (E6).

Eu aprendi que a gente acaba tendo outras maneiras de cuidar. Você sabe que não vai curar, então, a sua missão é dar apoio, conforto, é apoiar a família (E4).

A gente aprende a respeitar mais o ser humano, a saber ouvir mais, são esses os tipos de ensinamentos que eu tenho (T2).

O trecho do depoimento a seguir expõe alguns cuidados de enfermagem desenvolvidos com a criança hospitalizada com dor oncológica crônica.

A gente trabalha na avaliação e na parte da medicação da dor. Verificamos os sinais vitais, fazemos a higiene da criança, damos o banho ou a mãe é que dá o banho quando a criança é muito pequena e estranha a gente, nós também damos orientações com relação aos quimioterápicos (T6).

A subcategoria Revelando sofrimento familiar destaca que a hospitalização, o câncer e a dor crônica geram nos familiares da criança um intenso sofrimento e causam alterações na dinâmica e na estrutura da família.

A dor é muito forte para os pais. Nesse momento, muitas vezes, eles têm outros filhos em casa e o filho que ele deixou em casa, começa a desenvolver dor psicossomática e diz que está com dor de cabeça, mas na verdade, ele quer atenção. Há várias questões, há mudanças na estrutura familiar, a vida conjugal é alterada, o trabalho. Então, acaba sendo a dor da doença multiplicada (P6).

Quando eles (familiares) recebem o diagnóstico de câncer, eles ficam muito fragilizados e você tem que ter cuidado com a forma como você lida com eles (AS7).

O enfermeiro, nas relações de cuidado, percebe que o familiar apresenta necessidades de saúde de ordem afetiva, emocional e psicológica, conforme se observa nos trechos a seguir:

Eu observo o estado psicológico da mãe, como ela se encontra, oriento ela a dar um tempo se for preciso, trocar de acompanhante para buscar força (E1).

Eu percebo que eles (familiares) são muito carentes, carentes de abraço (E3).

Acho que a família precisa de muito apoio, até porque ela é a segurança daquela criança. Se os familiares não tiverem apoio, eles não conseguem dar apoio à criança (E4).

Nessa conjuntura, é importante destacar que o enfermeiro pode compor a rede social e de apoio da família da criança hospitalizada com dor oncológica crônica, nos diferentes contextos de saúde, favorecendo, inclusive, a continuidade do cuidado.

\section{DISCUSSÃO}

Nesta pesquisa, compreendeu-se que o contexto gerencial de cuidado à criança hospitalizada 
com dor oncológica crônica foi evidenciado como espaço permeado por aspectos objetivos e subjetivos, que de modo dialógico, ${ }^{9}$ tornam o cuidar uma experiência substancialmente complexa. Face ao exposto, o hospital foi caracterizado como um contexto de cuidado singular, posto que comporta especificidades assistenciais e gerenciais voltadas particularmente para os usuários que dependem dos serviços de hematologia e hemoterapia.

O contexto de cuidado é, também, marcado pela constante oferta de cursos de aperfeiçoamento profissional, os quais foram caracterizados pelos profissionais de enfermagem como necessários para uma assistência de qualidade à saúde da criança com doença hematológica. Nessa conjuntura, a dor oncológica crônica surge como condição contextual que interpela os profissionais de saúde a buscar as melhores evidências para a sua correta avaliação e manejo clínico.

Para este fim, os profissionais de saúde dispõem de um protocolo institucional elaborado para auxiliá-los na avaliação e no manejo da dor. O uso de protocolo no cuidado à criança com dor oncológica crônica foi caracterizado como essencial, visto que padroniza a analgesia farmacológica e confere praticidade ao desenvolvimento do cuidado. Estudo realizado em Nova Zelândia revelou que 97\% dos enfermeiros membros do College of Emergency Nurses consideraram importante ter um protocolo para nortear o gerenciamento da dor e caracterizaram como benéficos os cursos voltados para o gerenciamento desse fenômeno. ${ }^{16}$

A Unidade de Internação Pediátrica se caracteriza por ter crianças que dependem de uma abordagem multiprofissional permanente para manter qualidade de vida. No âmbito do trabalho em equipe, o enfermeiro diarista é o profissional que gerencia a Unidade de Internação Pediátrica, os recursos humanos de enfermagem e os recursos materiais. Além disso, todos os enfermeiros gerenciam o cuidado à criança hospitalizada com dor oncológica crônica.

Na perspectiva do gerenciamento do cuidado de enfermagem, uma revisão integrativa identificou oito ações do enfermeiro, quais sejam: dimensionar e liderar a equipe de enfermagem; planejar a assistência de enfermagem; educar/capacitar a equipe de enfermagem; gerenciar os recursos materiais; coordenar a realização dos cuidados; realizar o cuidado e/ou procedimentos mais complexos e avaliar os resultados das ações de enfermagem. ${ }^{17}$

O tratamento da criança com câncer foi caracterizado como longo e permeado por internações frequentes. Trata-se de uma experiência complexa, dolorosa, marcada por frequentes consultas médicas, quimioterapia, radiação, cirurgias, exames de rotinas, afastamento da escola, limitações para realizar algumas atividades, perda de oportunidades, mudanças na aparência e no estilo de vida. ${ }^{18-20}$ Nessa conjuntura, é necessário promover apoio e conforto, principalmente quando a criança se queixa de dor e apresenta comportamentos de medo e cansaço. ${ }^{21}$

A analgesia farmacológica com opioides se faz necessária para proporcionar o alívio da dor e atenuar o sofrimento infantil e familiar. Nessa conjuntura, a literatura ressalta a morfina como opioide que tem se mantido como primeira escolha para o tratamento da dor oncológica moderada a grave, por razões de familiaridade, disponibilidade, custo e superioridade. ${ }^{22} \mathrm{~A}$ escolha do medicamento é feita pela equipe médica, que no âmbito do trabalho em equipe, avalia a condição clínica da criança, define o tratamento e prescreve os medicamentos, seguindo o protocolo de dor definido pela instituição.

A dor oncológica crônica foi caracterizada pelos profissionais de enfermagem como forte, intensa e desesperadora. A hospitalização dessa criança é marcada por perdas e pela aquisição de novas limitações. Nesse sentido, um estudo revelou que a hospitalização da criança em condição crônica é extensa, complexa e marcada por várias transformações. A criança tem o seu corpo invadido por dispositivos que sinalizam suas limitações e suas novas demandas de cuidado. ${ }^{23}$

Um estudo ${ }^{24}$ apresentou que a internação foi significada pela criança oncológica como um processo necessário para o seu tratamento e para o controle de complicações relacionadas à doença e à quimioterapia, e por essa razão, ela aceita ir ao hospital sempre que necessário, mesmo que isso resulte em sua hospitalização. Na sua hospitalização, os sonhos e os projetos são desfeitos para dar lugar aos sentimentos de perda. ${ }^{25}$ Ressalta-se que perdas continuadas podem desencadear ansiedade e depressão na criança com câncer. ${ }^{26}$

A literatura ressalta que além de carregar o estigma do câncer, ela deixa de vivenciar situações importantes para o seu desenvolvimento humano, como por exemplo, a interação social com seus colegas e professores na escola. ${ }^{27}$ Ademais, o câncer é uma condição crônica de saúde que repercute na infância como uma condição limitadora para a prática de algumas brincadeiras. ${ }^{28}$

Nessa direção, um estudo ${ }^{29}$ registrou que o longo período de hospitalização pode levar a ocorrência de mudanças no envolvimento da criança 
com câncer em atividades lúdicas. Por essa razão, se ressalta que mesmo hospitalizada por conta da doença, a criança continua a se desenvolver como ser humano e precisa ser estimulada a brincar, a estudar, a questionar e a realizar tudo o que uma criança saudável é estimulada a fazer. ${ }^{27}$

Seu tratamento repercute no âmbito familiar, gerando, muitas vezes, alterações na dinâmica, estrutura e funcionalidade da família. No que concerne à funcionalidade desta, uma pesquisa apontou que famílias de crianças com dor crônica têm mau funcionamento familiar, apresentam mais conflitos e menos coesão quando comparadas às famílias de crianças saudáveis. ${ }^{30}$ Aliado ao exposto, um estudo revelou que o diagnóstico de câncer na criança gerou impactos negativos na qualidade de vida dos pais, pois $61 \%$ reportaram uma alimentação mais pobre, $69.9 \%$ relataram praticar menos exercícios, $81 \%$ reduziram seu período de sono e $82.9 \%$ referiram menos tempo para fazer atividades agradáveis. ${ }^{31}$

Além das desordens mencionadas, a hospitalização da criança com câncer leva a ocorrência de sofrimento familiar, o qual se inicia com o diagnóstico da doença. Essa circunstância inspira atenção da equipe multiprofissional de saúde para a possibilidade de oferecer suporte e apoio afetivo, emocional e social necessário para o enfrentamento da doença e do tratamento. Sobre essa circunstância, um estudo realizado em dois hospitais na Turquia identificou que todas as mães de crianças em tratamento de câncer referiram a necessidade de suporte social, mas apenas 73,9\% receberam o suporte necessário. A maioria do suporte social recebido foi emocional $(76,9 \%)$ e de informação $(49,2 \%)$, e 54,5\% das mães referiram esperar por suporte social vindo de enfermeiros. ${ }^{32}$

Ademais, destaca-se que, no âmbito familiar, irmãos de crianças hospitalizadas com câncer clamam por atenção dos pais e podem desenvolver dor psicossomática, a fim de tê-los ao seu lado. Diante disso, uma pesquisa ${ }^{33}$ revelou a importância de irmãos de crianças oncológicas serem apoiados e envolvidos no plano de cuidados no momento do diagnóstico da doença. Nessa mesma pesquisa, ${ }^{33}$ identificou-se que eles apresentaram necessidades de atenção, de informação, de ter seu medo/ preocupação gerenciado pela equipe, de querer ajudar e de ter uma rotina normal. Em linha com esse resultado, pesquisa ${ }^{34}$ reforça a importância de irmãos de crianças com câncer não serem negligenciados pelo enfermeiro, haja vista sua maior vulnerabilidade ao sofrimento psicológico.

O tratamento da criança com câncer visa alcançar a cura da doença, e quando isso não é possível, ele é gerenciado, principalmente, para proporcionar conforto e controle da dor. Nesse contexto, a atenção paliativa oncológica ganha ênfase e surge como uma abordagem que visa, inclusive, promover qualidade de vida à criança e prestar apoio à família nesse difícil momento. Compreende-se que o cuidado paliativo na infância é dirigido ao corpo, à mente e ao espírito, e envolve a prestação de apoio à família da criança. ${ }^{35}$ Desse modo, é uma abordagem que valoriza a vida e o viver com qualidade, mesmo na proximidade da morte.

A esse respeito, um estudo revelou que o cotidiano da criança em cuidados paliativos é bastante afetado pela dor, a qual foi apontada como causadora de sofrimento, especialmente quando gerenciada de forma precária pela equipe de saúde. ${ }^{36}$ Entende-se que a dor em crianças sob cuidados paliativos pode está relacionada à própria doença, intervenções médicas ou ao tratamento. ${ }^{37}$

O gerenciamento do cuidado de enfermagem à criança com dor crônica em atenção paliativa oncológica impõe pensar no conceito "dor total". A partir desse conceito, é possível compreender que a dor em pacientes com doença avançada é qualitativamente diferente da dor aguda, por ser multifatorial/multidimensional/complexa. Nessa perspectiva, a "dor total" é concebida quando ela transcende o seu aspecto físico e passa a envolver a dimensão espiritual, psicológica e social da pessoa, ameaçando a sua integridade. ${ }^{38}$

Com base no exposto, no âmbito da atenção paliativa oncológica, o cuidado à criança com dor crônica se revela como um convite para uma ruptura paradigmática, em busca de um pensamento que considere a relação dialógica entre a ordem/ desordem e a vida/morte. O princípio dialógico, na lente da complexidade, é o que comporta a ideia de união entre duas noções que deveriam excluirse reciprocamente, mas que, ao mesmo tempo, são complementares e antagônicas. ${ }^{9}$

Por esse olhar, cuidar de uma criança com dor crônica em atenção paliativa oncológica significa dar-lhe vida digna na proximidade da morte e respeitar a finitude humana na infinitude da vida. A relação dialógica morte/vida é interpretada na literatura como paradoxo da condição humana, pois se considera a morte como parte da vida e a vida como parte da morte. ${ }^{39}$ Logo, todo ser humano compartilha a ameaça da morte, ao passo que ela é parte integrante da vida. ${ }^{40}$

O gerenciamento do cuidado foi caracterizado pelos profissionais de enfermagem como difícil, visto que solicita conhecimento científico, técnico, sensibilidade e preparo emocional para lidar com 
o sofrimento da criança e do familiar. A literatura revela que o despreparo emocional e a dificuldade em lidar com a morte da criança e com o sofrimento de seus familiares se configuram como importantes causadores de estresse profissional, ${ }_{1}^{41}$ que, por sua vez, quando negligenciados, podem causar transtorno de ansiedade, depressão e absenteísmo.

Nessa direção, um estudo ${ }^{42}$ caracterizou o cuidado à criança oncológica como uma prática desafiadora, visto que o câncer é uma das doenças que mais leva à ocorrência de dor, sofrimento, medo, ansiedade e estresse, tanto para o paciente, como também para os familiares e para os profissionais de saúde.

Além de difícil, o cuidado à criança hospitalizada com dor oncológica crônica foi, também, caracterizado pelos profissionais de enfermagem como desgastante, visto que envolve todo um contexto marcado pela dialógica morte/vida, pelo sofrimento infantil e familiar, bem como pelo desafio em avaliar e realizar o manejo da dor oncológica crônica. Corroborando com esse resultado, um estudo $^{43}$ revelou, na perspectiva dos profissionais de enfermagem, que o cotidiano de cuidado à criança com câncer se configura como uma experiência desgastante e triste. Nessa conjuntura, os participantes do estudo supracitado ${ }^{43}$ referiram que o mais difícil é a relação de envolvimento diante do sofrimento das crianças e dos pais.

O cuidado à criança hospitalizada com dor crônica oncológica foi caracterizado pelos profissionais de enfermagem como uma experiência permeada por aprendizagem, posto que há descobertas sobre novas modalidades de cuidado e valores humanos. Sob essa perspectiva, a literatura ${ }^{44}$ registra que profissionais de enfermagem que atuam em unidade de oncologia pediátrica aprendem, com a experiência do cuidado, a ressignificar a vida, as relações familiares/profissionais, a doença e a morte. ${ }^{44}$

Entre os cuidados de enfermagem desenvolvidos à criança hospitalizada com dor oncológica crônica, constam: administração de medicamentos, verificação de sinais vitais, cuidados de higiene, avaliação e manejo da dor e orientações sobre o tratamento. Corroborando com esse resultado, a literatura ${ }^{45-46}$ revela que a criança hospitalizada com câncer necessita de múltiplos cuidados, entre os quais: administração de medicamentos, cuidados de higiene, punção venosa periférica, verificação dos sinais vitais, administração de quimioterápicos, transfusão sanguínea, treinamento com o familiar para a realização dos cuidados, promoção de conforto, apoio à família, avaliação e manejo da dor.
Os resultados desse estudo apresentam limitações quanto ao critério de generalização teórica, visto que revelam aspectos contextuais referentes a uma única instituição do município do Rio de Janeiro. No entanto, é possível que ele apresente uma certa proximidade com outras realidades de cuidado à criança hospitalizada com dor oncológica crônica. Assim, recomenda-se o desenvolvimento de novas pesquisas sobre o tema, a fim de tornar mais denso o conteúdo produzido.

\section{CONCLUSÃO}

A partir dos resultados apresentados, compreendeu-se que o contexto de cuidado à criança hospitalizada com dor oncológica crônica é complexo, pois além de solicitar dos profissionais de saúde conhecimento técnico, científico, sensibilidade e preparo emocional, a dialógica morte/vida se apresenta como condição que os interpela a reconhecer e a lidar com a finitude da vida. A experiência do cuidar de uma criança em condição de sofrimento, causada, sobremodo, pela dor oncológica crônica, se revela como difícil e desgastante, contudo, é permeada por aprendizagem de âmbito pessoal e profissional.

A hospitalização da criança com dor oncológica crônica é longa, marcada por perdas, limitações, alterações na dinâmica familiar. Seus impactos podem, também, incidir sobre a estrutura e funcionalidade familiar, o que pode tornar o enfrentamento da doença uma experiência de ordem ou desordem na família. Nessa conjuntura, é importante destacar que a dor oncológica crônica foi caracterizada pelos profissionais de saúde como forte, intensa e desesperadora, requerendo, por parte destes, tratamento farmacológico adequado à base de opioides.

O cuidado paliativo emerge, no contexto oncológico, como um caminho para a promoção do conforto, controle da dor e apoio à família, a fim de preservar a integridade da criança e de oferecer o suporte multidimensional que a família necessita. Nessa conjuntura, é importante que o gerenciamento do cuidado de enfermagem seja realizado de modo coerente com a natureza multifatorial da dor, como também com as demandas multidimensionais da criança e do familiar.

Destaca-se a atuação do enfermeiro no contexto oncológico pediátrico, cujas demandas estão relacionadas ao gerenciamento da unidade de internação pediátrica, gerenciamento de recursos humanos e materiais, bem como ao gerenciamento do cuidado direto à criança. Para tanto, a disposição 
de protocolos de dor e a participação em cursos de capacitação para adequada avaliação e manejo clínico surgem como aspectos contextuais necessários para a efetividade do gerenciamento do cuidado.

\section{REFERÊNCIAS}

1. Instituto Nacional de Câncer José Alencar Gomes da Silva (INCA). Incidência, mortalidade, morbidade hospitalar por câncer em crianças, adolescentes e adultos jovens no Brasil: informações dos registros de câncer e do sistema de mortalidade [Internet]. 2016 [cited 2017 Feb 23]. Available from: http://www1. inca.gov.br/inca/Arquivos/incidencia-mortalidademorbidade-hospitalar-por-cancer.pdf

2. Reis RS, Santos MO, Camargo B, Oliveira JFP, Thuler LCS, Pombo-de-Oliveira MS. Early childhood leukemia incidence trends in Brazil. Pediatr Hematol Oncol [Internet]. 2016 [cited 2017 Sep 08]; 33(2):83-93. Available from: https://www.ncbi.nlm.nih.gov/ pubmed/26925506

3. Ward E, De Santis C, Robbins A, Kohler B, Jemal A. Childhood and adolescent cancer statistics, 2014. CA Cancer J Clin [Internet]. 2014 [cited 2017 Feb 22]; 64(2):83-103. Available from: https:/ / www.ncbi.nlm. nih.gov/pubmed/24488779

4. Van Cleve L, Muñoz CE, Savreda M, Riggs M, Bosset E, Grant M, et al. Symptoms in children with advanced cancer. Cancer Nurs [Internet]. 2012 [cited 2017 Feb 22]; 35(2):115-25. Available from: https:/ / www.ncbi. nlm.nih.gov/pubmed/21760496

5. Fortier MA, Wahi A, Bruce C, Maurer EL, Stevenson R. Pain Management at home in children with cancer: a daily diary study. Pediatr Blood Cancer [Internet]. 2014 [cited 2017 Feb 22]; 61(6):1029-33. Available from: https://www.ncbi.nlm.nih.gov/pubmed/24376073

6. Olagunju AT, Sarimiye FO, Olagunju TO, Habeedu MYM, Aina OF. Child's symptom burden and depressive symptoms among caregivers of children with cancers: an argument for early integration of pediatric palliative care. Ann Palliat Med [Internet]. 2016 [cited 2017 Sep 08]; 5(3):157-65. Available from: https://www.ncbi.nlm.nih.gov/pubmed/27199271

7. World Health Organization (WHO). WHO guidlines on the pharmacological treatment of persisting pain in children with medical illnessess [Internet]. 2012 [cited 2017 Feb 22]. Available from: http:/ / whqlibdoc.who. int/publications/2012/9789241548120_Guidelines. pdf

8. American Pain Society (APS). Assessment and management of children with chronic pain. [Internet]. 2012 [cited 2017 Feb 22]. Available from: http:/ / www. americanpainsociety.org/uploads/pdfs/aps12-pcp. pdf

9. Morin E. O método 5: a humanidade da humanidade. $5^{\mathrm{a}}$ ed. Porto Alegre (RS): Sulina; 2012.

10. Afshan G, Bashir K. Cancer pain in children: a two- step strategy. Anaesthesia Pain \& Intensive Care [Internet]. 2014 [cited 2017 Feb 22]; 18(1):106-10. Available from: http://www.apicareonline.com/ special-article-cancer-pain-in-children-a-two-stepstrategy /

11. Batalha LMC, Fernandes AM, Campos C, Gonçalves AMPMPC. Pain assessment in children with cancer: a systematic review. J Nurs Ref [Internet]. 2015 [cited 2017 Feb 22]; 4(5):119-27. Available from: https:/ / rr.esenfc.pt/rr/index.php? module $=$ rr\&target $=$ pu blicationDetails\&pesquisa $=\& i d$ artigo $=2516 \&$ id revista $=24 \&$ id_edicao $=78$

12. Jones P, Blunda M, Biegel B, Carlson LE, Biel M, Wiener L. Can mindfulness-based interventions help adolescents with cancer? Psychooncology [Internet]. 2013 [cited 2017 Feb 22]; 22(9):2148-51. Available from: https://www.ncbi.nlm.nih.gov/pubmed/23417883

13. Neale KL. The fifth vital sign: chronic pain assessment of the adolescent oncology patient. J Pediatr Oncol Nurs [Internet]. 2012 [cited 2017 Feb 22]; 29(4):18598. Available from: https:/ / www.ncbi.nlm.nih.gov/ pubmed/22797680

14. Tarozzi, M. O que é a grounded Theory: metodologia de pesquisa e de teoria fundamentada nos dados. Petrópolis (RJ): Vozes; 2011.

15. Strauss AL, Corbin J. Pesquisa qualitativa: técnicas e procedimentos para o desenvolvimento de teoria fundamentada. $2^{a}$ ed. Porto Alegre (RS): Artmed; 2008.

16. Petrorius A, Searle J, Marshall B. Barriers and enablers to emergency departament nurses' management of patients' pain. Pain Manag Nurs [Internet]. 2015 [cited 2017 Feb 22]; 16(3):372-9. Available from: https:// www.ncbi.nlm.nih.gov/pubmed/25440235

17. Santos JLG, Pestana AL, Guerrero P, Meirelles BSH, Erdmann AL. Nurses'practices in the nursing and health care management: integrative review. Rev Bras Enferm [Internet]. 2013 [cited 2017 Feb 22]; 66(2):257-63. Available from: http:/ / www.scielo.br/scielo.php?script=sci_arttext\&pid =S0034-71672013000200016

18. Gray WN, Szulczewski LJ, Regan SM, Williams JA, Pai AL. Cultural influences in pediatric cancer from diagnosis to cure/end of life. J Pediatr Oncol Nurs [Internet]. 2014 [cited 2017 Feb 22]; 31(5):252-71. Available from: https://www.ncbi.nlm.nih.gov/ pubmed/25299000

19. Battista AD, Dupuis LL, Cassidy M, Portwine C, Johnston DL, Silva MP, et al. Parent attributions about child symptoms related to cancer therapy. J Pediatr Oncol Nurs [Internet]. 2016 [cited 2017 Sep 08]; pii:104345215628002. Available from: https:// www.ncbi.nlm.nih.gov/pubmed/26825448

20. Popp JM, Conway M, Pantaleao A. Parents' experience with their child's cancer diagnosis: do holpefulness, family functioning, and perceptions of care matter? J Pediatr Oncol Nurs [Internet]. 2015 [cited 2017 Feb 22]; 32(4):253-60. Available from: https:// www.ncbi. 
nlm.nih.gov/pubmed/25556107

21. Brannstrom CA, Norberg A. Children undergoing cancer treatment describe their experiences of comfort in interviews and drawings. J Pediatr Oncol Nurs [Internet]. 2014 [cited 2017 Feb 22]; 31(3):135-46. Available from: https:/ / www.ncbi.nlm.nih.gov/ pubmed/24651546

22. Caraceni A, Hanks G, Kaasa S, Bennett MI, Brunelli C, Cherny N, et al. European Palliative Care Research Collaborative (EPCRC). Use of opioid analgesics in the treatment of cancer pain: evidence-based recommendations from the EAPC. Lancet Oncol [Internet]. 2012 [cited 2017 Feb 22]; 13(2):58-68. Available from: https://www.ncbi.nlm.nih.gov/ pubmed/22300860

23. Silva TP, Silva MM, Silva LJ, Silva IR, Leite JL. Contextual especificities of nursing care to hospitalized children with chronic condition. Cienc Cuid Saude [Internet]. 2015 [cited 2017 Feb 22]; 14(2):1082-90. Available from: http:/ / periodicos.uem.br/ojs/index. php/CiencCuidSaude/article/view/23814/14719

24. Gomes IP, Lima KA, Rodrigues LV, Lima RAG, Collet N. From diagnosis to survival of pediatric cancer: children's perspective. Texto Contexto Enferm [Internet]. 2013 [cited 2017 Feb 22]; 22(3):6719. Available from: http://www.scielo.br/pdf/tce/ v22n3/en_v22n3a13.pdf

25. Sousa MLXF, Reichert APS, Sá LD, Assolini FEP, Collet N. Stepping into a new world: the meaning of sicken for the child with cancer. Texto Contexto Enferm [Internet]. 2014 [cited 2017 Feb 22]; 23(2):3919. Available from: http://www.scielo.br/pdf/tce/ v23n2/pt_0104-0707-tce-23-02-00391.pdf

26. Nikfarid L, Rassouli M, Borimnejad L, Alavimajd H. Chronic sorrow in mothers of children with cancer. J Pediatr Oncol Nurs [Internet]. 2015 [cited 2017 Feb 22]; 32(5):314-9. Available from: https://www.ncbi. nlm.nih.gov/pubmed/25576316

27. Viero V, Beck CLC, Coelho APF, Lima SBS, Machado BP. Coping of children with cancer on school removal because of hospitalization. Rev Enferm UFSM [Internet]. 2014 [cited 2017 Feb 22]; 42(2):368-77. Available from: https:/ / periodicos.ufsm.br/reufsm/ article/view/10956/pdf

28. Silva LF, Cabral IE. Cancer repercussions on play in children: implications for nuring care. Texto Contexto Enferm [Internet]. 2014 [cited 2017 Feb 22]; 23(4):93543. Available from: http://www.scielo.br/pdf/tce/ v23n4/pt_0104-0707-tce-23-04-00935.pdf

29. Silva LF, Cabral IE. Rescuing the pleasure of playing of child with cancer in a hospital setting. Rev Bras Enferm [Internet]. 2015 [cited 2017 Feb 22]; 68(3):3917. Available from: http:/ / www.scielo.br/pdf/reben/ v68n3/0034-7167-reben-68-03-0391.pdf

30. Palermo TM, Valrie CR, Karlson CW. Family and parent influences on pediatric chronic pain: a developmental perspective. Am Psychol [Internet].
2014 [cited 2017 Feb 22]; 69(2):142-52. Available from: https://www.ncbi.nlm.nih.gov/pubmed/24547800

31. Wiener L, Viola A, Kearney J, Mullins LL, ShermanBien S, Zadeh S, et al. Impact of caregiving for a child with cancer on parenteral health behaviours, relationship quality, and spiritual faith: do lone parents fare worse? J Pediatr Oncol Nurs [Internet]. 2015 [cited 2017 Sep 08]; 33(5)378-86. Available from: https://www.ncbi.nlm.nih.gov/pubmed/26668211

32. Atlay N, Kilicarslan E, Sari Ç, Kisecik Z. Determination of social support needs and expectations of mothers of children with cancer. J Pediatr Oncol Nurs [Internet]. 2014 [cited 2017 Feb 22]; 31(3):147-53. Available from: https://www.ncbi.nlm.nih.gov/pubmed/24647009

33. O'Shea ER, Shea J, Robert T, Cavanaugh C. The needs of siblings of children with cancer: a nursing perspective. J Pediatr Oncol Nurs [Internet]. 2012 [cited 2017 Feb 22]; 29(4):221-31. Available from: https://www.ncbi.nlm.nih.gov/pubmed/22797684

34. Zegaczewski T, Chang K, Coddington J, Berg A. Factors related to healthy siblings psychosocial adjustment to children with cancer: an integrative review. J Pediatr Oncol Nurs [Internet]. 2015 [cited 2017 Sep 08]; 33(3):218-27. Available from: https:/ / www.ncbi.nlm.nih.gov/pubmed/26483426

35. World Health Organization (WHO). Global atlas of palliative care at the end of life [Internet]. 2014 [cited 2017 Feb 22]. Available from: http:/ / www.who.int/ nmh/Global_Atlas_of_Palliative_Care.pdf

36. Borghi CA, Rossato LM, Damião EB, Guedes DM, Silva EM, Barbosa SM, et al. Living with pain: the experience of children and adolescents in palliative care. Rev Esc Enferm USP [Internet]. 2014 [cited 2017 Feb 22]; 48(esp):68-74. Available from: http:/ / www. scielo.br/pdf/reeusp/v48nspe/pt_0080-6234-reeusp48-esp-068.pdf

37. Downing J, JassalSS, Mathews L, Brits H, Friedrichsdorf SJ. Pediatric pain management in palliative care. Pain Manag [Internet]. 2015 [cited 2017 Sep 08]; 5(1):23-35. Available from: https://www.ncbi.nlm.nih.gov/ pubmed/25537696

38. Rodrigues AJ, Bushatsky M, Viaro WD. Palliative care in children with cancer: integrative review. J Nurs UFPE on line [Internet]. 2015 [cited 2017 Sep 08]; 9(2):718-30. Available from: http:/ / www.revista.ufpe. br/revistaenfermagem/index.php/revista/article/ view/7129/pdf_7201

39. Mariotti H. Pensando diferente: para lidar com a complexidade, a incerteza e a ilusão. São Paulo (SP): Atlas; 2010.

40. Souza e Souza LP, Ribeiro JM, Rosa RB, Gonçalves RCR, Silva CSO, Barbosa DA. Death and the dying process feelings expressed by nurses. Enferm Global [Internet]. 2013 [cited 2017 Sep 08]; 12(32):230-37. Available from: http://scielo.isciii.es/pdf/eg/ v12n32/pt_administracion4.pdf

41. Silva MM, Vidal JM, Leite JL, Silva TP. Estratégias 
de cuidados adotados por enfermeiros na atenção à criança hospitalizada com câncer avançado e no cuidado de si. Cienc Cuid Saude [Internet]. 2014 [cited 2017 Feb 22]; 13(3):471-8. Available from: http:// periodicos.uem.br/ojs/index.php/CiencCuidSaude/ article/view/19937

42. Reis TLR, Paula CC, Potrich C, Padoin SMM, Bin A, Mutti CF, Bubadué RM. Relações estabelecidas pelos profissionais de enfermagem nos cuidados às crianças com doença oncológica avançada. Aquichan [Internet]. 2014 [cited 2017 Feb 22]; 14(4):496-508. Available from: http:/ /aquichan.unisabana.edu.co/ index.php/aquichan/article/view/3070/pdf

43. Mutti CF, Padoim SMM, Paula CC. Spaciality of beingnursing-professional in the world of caring to children who has cancer. Esc Anna Nery Rev Enferm [Internet]. 2012 [cited 2017 Feb 22]; 16(3):493-9. Available from: http://www.scielo.br/pdf/ean/v16n3/10.pdf
44. Viero V, Beck CLC, Coelho APF, Pai DP, Freitas PH, Fernandes MNS. Pediatric oncology nursing workers: the use of defensive strategies at work. Esc Anna Nery [Internet]. 2017 [cited 2017 Sep 08]; 21(4):e20170058. Available from: http://www.scielo.br/pdf/ean/ v21n4/1414-8145-ean-2177-9465-EAN-2017-0058.pdf

45. Silva TP, Silva MM, Valadares GV, Silva IR, Leite JL. Nursing care management for children hospitalized with chronic conditions. Rev Bras Enferm [Internet]. 2015 [cited $2017 \mathrm{Feb} 22$ ]; 68(4):556-63. Available from: http://www.scielo.br/pdf/reben/v68n4/en_00347167-reben-68-04-0641.pdf

46. Silva TP, Leite JL, Santos NLP, Silva IR, Mendonça ACA, Santos MJM, et al. Nursing care to the child with cancer: an integrative review of the literature. Rev Enferm UFSM [Internet]. 2013 [cited 2017 Feb 22]; 3(1):68-78. Available from: https:// periodicos.ufsm. $\mathrm{br} /$ reufsm/article/view/6918 